wie in den USA ,erst in ihrer Anwendung ihrer Ausprägung finden" werde (S. 288) - einer allzu engen Anlehnung an das Muster aus Übersee sind (vielmehr) einfach-gesetzliche, verfassungs- und wohl auch, was Eisenblätter leider kaum erörtert, europarechtliche Schranken gesetzt.

Die Untersuchung ist durchweg anregend und informativ, auch wenn das Verhältnis von Ökonomie und Recht zuweilen eher additiv und nicht optimal wechselseitig verknüpft erscheint. Ein inhaltliches Manko mag man darin sehen, dass Eisenblätter den föderalen Aspekt allenfalls streift, obgleich auch hier - in puncto Regulierungsbehörde und -gesetz - der Blick auf Parallelen und Unterschiede zwischen zwei Bundesstaaten aufschlussreich hätte sein können. Gewichtiger erscheint der Einwand, dass sie offenbar das vom Regulierer verfolgte „öffentliche Interesse“ („public interest“) des Öfteren in einen Gegensatz zum - hier wie dort demokratischen! - politischen Prozess bringt und (damit) Unabhängigkeit als bloßen Sachverstand (miss-)versteht, ohne das Problem der Akzeptanz und der Legitimität aufzuwerfen. Zudem spart die Autorin die (zumindest partielle) Verwandtschaft der deutschen Regulierung im TK- und im Postsektor zu speziellen Instrumentarien der Wirtschaftsaufsicht (insbesondere über Finanzmärkte) fast durchweg aus. Speziell die Lizenzierungsvorschriften ( $\$ \int 6 \mathrm{ff}$. TKG) können jedoch ihre Herkunft aus dem Gewerbe(polizei)recht nicht verleugnen, selbst wenn sie auch einen gleitenden Übergang der früheren (Netz- und Telefondienst-)Monopole in künftige schiere Überwachung (mit einem staatlichen Eingreifen allein ex post) intendier(t)en. Endlich sollte sich eine Arbeit über Regulierung in der „Telekommunikation" auch etwas eingehender mit - im TKG (\$S 85 ff.) durchaus ausführlich behandelten - Spezifika des Mediums, nämlich der (gebotenen) Vertraulichkeit der Kommunikation (einschließlich des Schutzes der informationellen Selbstbestimmung) befassen; spätestens im Blick auf Art. 10 GG zeigt sich, dass der Telekommunikations- eben kein $\mathrm{x}$-beliebiger Markt ist. Die immer wieder erhobenen Forderungen nach Abbau der (asymmetrischen) Regulierung dürften daher nicht allein sozialstaatliche Belange gering schätzen, sondern auch spezifische grundrechtliche Schutzpflichten verkennen, wenn sie denn nicht allein auf die (in der Tat schon rein praktisch höchst diffizi- le) Preisregulierung abzielen. Da hiervon allein marktbeherrschende Unternehmen betroffen sind, scheint sich die Debatte ohnehin zunehmend auf die Frage der richtigen Abgrenzung der sachlich und/oder räumlich relevanten Märkte zu verlagern. Auch dies belegt, dass anders als dies wohl Eisenblätter sieht - Regulierung in der Telekommunikation (wie im Postsektor) in Deutschland ein Amalgam aus Gewerbe-, Kartell- und Privatisierungsfolgenrecht bildet, wobei nur der letzte Bestandteil temporärer Art sein dürfte.

Nur schade, dass die Arbeit über kein Sach/Personenverzeichnis verfügt, das ihren Ertrag besser erschließt.

Ludwig Gramlich

\section{Jens Wolling}

\section{Politikverdrossenheit durch Massenmedien?}

Der Einfluss der Medien auf die Einstellungen der Bürger zur Politik

Wiesbaden: Westdeutscher Verlag, 1999. - 287 $\mathrm{S}$.

\section{ISBN 3-531-13404-3}

Politikverdrossenheit ist der populäre Begriff für ein Syndrom verschiedener Einstellungen der Bürgerinnen und Bürger zum politischen System, seinen Strukturen und dem Handeln der politischen Akteure. In der Politikwissenschaft erlebt das Thema seit einigen Jahren eine gewisse Konjunktur. Da wird über Begrifflichkeit, Messung und Messbarkeit, über Ursachen und Folgen diskutiert. Wenn nach den Ursachen gefragt wird, beweist die Politikwissenschaft indessen einmal mehr einen ihrer blinden Flecken. Zum einen verschließt sie sich der Tatsache, dass die Bevölkerung Politik überwiegend in der Vermittlung der Massenmedien wahrnimmt und diese daher bei der Suche nach den Auslösern von Politikverdrossenheit oder als intervenierende Variable unbedingt $\mathrm{zu}$ berücksichtigen sind. Daraus folgt dann zum anderen, dass der diesbezügliche Forschungsstand, den es in der Kommunikationswissenschaft gibt, ebenfalls kaum herangezogen wird.

Im Zentrum der Studie von Jens Wolling, und das sagt ja auch der Titel des Buches (was übrigens immer weniger selbstverständlich ist), steht eben die Frage nach dem Einfluss der Medien auf Politikverdrossenheit. So weit wie 
möglich repliziert er damit eine Untersuchung der Rezensentin, die - damals inspiriert durch die weit umfangreichere, aber gewiss nicht übertragbare US-amerikanische Forschung zu diesem Thema - Ende der achtziger Jahre durchgeführt wurde. Nach mehreren kleineren Studien, die alle auf Sekundäranalysen beruhen, ist Wollings Arbeit die erste größere Untersuchung, die das duale Rundfunksystem berücksichtigt. Das ist vor allem deshalb wichtig, weil durch die Veränderungen zumal der politischen Angebote im dualen System - negative Auswirkungen auf die Einstellungen der Bevölkerung zur Politik erwartet wurden.

Wolling leitet sein Buch ein mit einer Diskussion verschiedener Dimensionen von Politikverdrossenheit. Dafür sichtet er zunächst die vorliegenden, überwiegend aus den USA stammenden theoretischen Konzepte und empirische Indikatoren. Später systematisiert er diese, indem er sie den drei Dimensionen der Politik (policy, polity, politics) zuordnet. Obwohl, wie Wolling selber schreibt, diese Unterteilung nicht ganz trennscharf ist, gelingt die danach vorgenommene Systematisierung recht gut und kann im Übrigen auch dazu dienen, nach der Sichtung des Forschungsstandes Forschungslücken zu identifizieren.

Das Fazit nach der Auseinandersetzung mit deutschen Untersuchungen, die dem Zusammenhang zwischen Mediennutzung und Einstellungen zur Politik nachgegangen sind, muss kritisch ausfallen. Die wenigen Studien, die zu diesem Thema vorliegen, sind heterogen in ihrer Vorgehensweise, die Variablen, die verwendet werden, wechseln und sind oft nicht genügend differenziert; schließlich werden Analysen vorgelegt, die auf den Einsatz multivariabler Verfahren verzichten, obwohl das bei einem so komplexen Beziehungsgeflecht relevanter Faktoren unerlässlich ist.

Wollings eigene Untersuchung, die im zweiten Teil des Buches präsentiert wird, basiert im Wesentlichen auf einer Bevölkerungsbefragung in Dresden (1996) sowie einer Inhaltsanalyse von Fernsehnachrichtensendungen und Tageszeitungen (1996). Ergänzend wurde eine Sekundäranalyse von drei repräsentativen Bevölkerungsbefragungen $(1995,1996)$ vorgenommen. Indem Wolling Umfrage und Inhaltsanalyse verbindet, also nicht nur Variablen der Mediennutzung mit Einstellungen zur Politik zusammenführt, trägt er einem weiteren Kritikpunkt Rechnung, der sich auf die bisherige Forschung richtet. Vermutungen über einen Einfluss der Medien auf politische Einstellungen betreffen eigentlich immer die Angebote, die genutzt werden, kaum eine Untersuchung hat aber bisher tatsächlich diese Angebote analysiert, sondern lediglich mit Nutzungsvariablen - mehr oder weniger plausible - Vermutungen über die genutzten Inhalte verbunden.

Aus der Vielzahl der Detailergebnisse seien hier nur ein paar herausgegriffen. Erstens: Für die Videomalaise-Hypothese, die in den siebziger Jahren aus den USA kam und die dem Fernsehen, und zwar spezifisch der Nutzung informierender Angebote, einen negativen Effekt zuschreibt, gibt es hier keine Unterstützung. Zweitens: Wie schon in früheren Untersuchungen, zeigen sich Zusammenhänge zwischen der Nutzung von unterhaltenden Medienangeboten und politischen Einstellungen. Aber: Hier geht intensiver Konsum unterhaltender $\mathrm{Me}$ dien und Inhalte mit einem positiveren Politikbild einher. Nur bezüglich der eigenen Einflussüberzeugung gibt es einen negativen Zusammenhang zur Nutzung unterhaltender Zeitungsinhalte. Drittens: Die persönlichen Erfahrungen der Rezipienten spielen eine wichtige Rolle für die Einstellungen zur Politik. Diese sollten also mit berücksichtigt werden, wenn wir Politikverdrossenheit untersuchen. Allerdings gibt es dabei aber wohl auch zu bedenken, dass sich das, was als unmittelbare Erfahrung bezeichnet wird - nämlich, wie in dieser Studie, Beurteilung der Wirtschaft oder Defizite der Politik - mit Eindrücken und Bewertungen vermischt, die aus den Medien übernommen werden. Das zeigt sich wohl auch darin und damit wird ein vierter Befund herausgegriffen -, dass gerade die Wirtschaftsberichterstattung in den Medien Zusammenhänge zu den politischen Einstellungen aufweist.

Insbesondere mit seinen konzeptionellen Überlegungen hat Wolling neue Perspektiven für die weitere Forschung in diesem Bereich eröffnet, und methodisch hat er mit seinem konsequent (und notwendigerweise) multivariaten Vorgehen Maßstäbe gesetzt. Wenn es an der Studie etwas zu kritisieren gibt, ist es ihre Beschränkung auf eine ostdeutsche Großstadt. Wir wissen, dass das Mediennutzungsverhalten ebenso wie die hier untersuchten Einstellungen zur Politik in den ostdeutschen Bundesländern zum Teil deutlich anders sind als im Westen. Die vorliegenden Befunde zu verallgemeinern, 
ist also problematisch; Wolling selbst scheint das gelegentlich zu vergessen.

Christina Holtz-Bacha

\section{Bart Pattyn (Ed.)}

\section{Media ethics}

Opening social dialogue

Leuven: Peeters, 2000. - 422 S.

ISBN 90-42909-02-1

Der Band resultiert aus dem „Core Materials Project“ zur Entwicklung von Basismaterial für die berufsethische Ausbildung, das vom „European Ethics Network“ betrieben und von der Europäischen Kommission finanziell unterstützt wird. Johan Verstraeten formuliert im Vorwort die Prämissen des Projekts zur Berufsethik im Allgemeinen: Sie setze das Verständnis der Aufgabe und des sozialen Kontextes des jeweiligen Berufes voraus; es gebe keine neutrale Berufsethik, sie sei stets von spezifischen Kulturtraditionen beeinflusst; berufsethische Bildung bedeute vor allem ein Training in gesellschaftlicher Verantwortung, ein Bewusstmachen der sozialen Folgen des beruflichen Handelns; und berufsethische Bildung müsse mit der Entwicklung zur mündigen Person einhergehen. Zusammengefasst sei berufsethische Bildung ein globales pädagogisches Vorhaben, in dem es nicht nur um die Vermittlung von ethischen Kenntnissen durch Spezialisten, sondern auch um deren praxisnahes Erproben in einem sozialen Zusammenhang gehe, in dem Berufsethos vorgelebt wird. Entsprechend wenden sich die aus dem Projekt hervorgehenden Materialien zugleich an Ethiker und an Praktiker auf verschiedenen Gebieten, in diesem Fall an die Professionellen der Medien und auch an deren Nutzer.

Über die allgemeinen Prämissen hinaus erläutert Herausgeber Bart Pattyn in der Einleitung medienspezifische Aspekte, die den Texten des Bandes gemeinsam sind. Dass die normative Basis der Medienethik mit der Idee des demokratischen Dialogs, des offenen gesellschaftlichen Gesprächs innerhalb von Kulturen oder zwischen ihnen korrespondiert, lassen tatsächlich alle Beiträge erkennen. Besonders wichtig erscheint Pattyns Hinweis, dass für die Beurteilung von Medien weniger wichtig ist, was sie ans Licht bringen, als was sie verbergen.
Daraus kann man eine professionelle Grundpflicht zum Publizieren ableiten.

Entsprechend dem in Vorwort und Einleitung formulierten Programm ist der Band in vier Abschnitte gegliedert: Mit Geschichte und philosophischen Grundlagen der Medienethik befassen sich Clifford G. Christians, Robert A. White, Kaarle Nordenstreng, Michele Nicoletti und Cees J. Hamelink. Den kulturellen und ökonomischen Kontext fassen Luc Van Poecke, Walter Lesch, Bart Pattyn und Hilde Van den Bulck ins Auge. Es folgt der Abschnitt, der dem Praxisanspruch am meisten gerecht wird, nämlich die Ethik der professionell in den Medien Tätigen. In diesem zentralen Teil schreibt Barbara Thomaß über das journalistische Berufsethos; Huub Evers problematisiert medienethische Kodizes und diskutiert deren Vor- und Nachteile; Robert A. White stellt Thesen darüber auf, woran ein ethisch einwandfrei handelnder öffentlicher Kommunikator zu erkennen ist, und erinnert in diesem $\mathrm{Zu}$ sammenhang an die Qualität der demokratischen Kommunikation; Cees J. Hamelink bringt die Medienethik mit dem Problem der moralischen Wahlfreiheit in Zusammenhang; Grundzüge einer Ethik des Umgangs mit Computern skizziert Porfirio Barroso; mit den Mischformen der Marketing-Kommunikation und dem Verschwinden der Grenze zwischen Information und Geschäft befassen sich Aagje Geerardyn und Guido Fauconnier; Marcel Becker problematisiert den Vormarsch des Privaten in der Öffentlichkeit und fragt, was er mit der journalistischen Freiheit zu tun hat; und Barbara Thomaß schließlich vergleicht in ihrem zweiten Beitrag, mit welchen Inhalten und nach welchen Methoden journalistische Berufsethik in den drei Ländern Deutschland, Frankreich und Großbritannien gelehrt wird, wobei sie auf eine mehr oder weniger repräsentative Umfrage an Journalistenschulen zurückgreift. Im vierten großen Abschnitt befassen sich Cees J. Hamelink und Rüdiger Funiok mit einem relativ selten behandelten Thema, nämlich der Ethik der Mediennutzung.

Stärken und Schwächen des Bandes sind angesichts der internationalen Autorenschaft erwartbar, wobei ich nicht auf Einzelheiten eingehen kann. Ein großer Gewinn für den Leser liegt darin, dass er über den engen Horizont der medienethischen Debatte im eigenen Land hinauszuschauen lernt. Kaarle Nordenstrengs Beitrag beispielsweise listet Basisinformationen 